\title{
Final discussion
}

DR. WHITE With regard to the uneven distribution of radioactive elements in these joints, it occurs to me that in all the scans we are seeing a two-dimensional view of something three-dimensional. I wonder if this alleged maldistribution may not be seen, because you are looking at several layers upon which you have a quite even distribution of material, and if the villae which have been described, and indeed the cysts and geodes, may in fact be the cause of this.

DR. VEALL That seems a perfectly reasonable suggestion.

DR. ANSELL There were one or two autoradiographs that we did years ago which suggested a varying uptake of the radioactive material, particularly if there was a lot of fibrin about. The distribution in the joint showed that the ${ }^{198} \mathrm{Au}$ went on to the fibrin and, if there was a fibrin layer, you might find a lot of yttrium or gold at that point on the membrane but not much further on where the synovial membrane was thinner.

I should like to bring up another point that perhaps some workers would comment on. These days we are constantly talking about the results of surgical synovectomy and the results of radiation synovectomy. Dr. Gumpel did indicate that it was important to take into consideration the patient's general disease activity and state. This is one of the extremely difficult points in assessing the results in trials, to know how far the overall activity of the disease affects the results. Does the patient with the most actively inflamed synovial membrane have a greater leakage and therefore fail to have a good result because more of the radio-isotope has been lost? Is it the patient with the mildest synovitis who does best?

DR. GUMPEL This is a very difficult question, and since no-one else is rushing to answer it, I can only base my answer on a very small number of patients. Certainly, among the patients whose results Dr. Williams has shown you this morning, those with the biggest leak are also those who had a poor result, and subsequently did well with chrysotherapy. I meant to say in my talk that some of these patients later experienced a generalized flare-up of disease activity and were therefore given gold or penicillamine. As Dr. Ansell says, this is one of the most difficult areas of interpretation: how does a poor result become, by the addition of gold or penicillamine, an excellent result? Only one of the patients I am thinking of had had a relapse in terms of the knee which had been treated with radiation synovectomy.

DR. ROBERTS Our figures were certainly very much the same as the results from surgical synovectomy. It is exactly as you say; the patients with the mild disease do well, and these who had advanced disease and advanced radiological changes show subsequent but normal deterioration.

DR. ANSELL The degree of disease activity is very important. I am currently reviewing some juvenile synovectomies of the knee, and these can from one year to another convert from a bad to a good result when the general disease activity declines. We must bear this in mind when assessing long-term results, and be particularly critical of this. Dr. Gumpel asked a question about psoriatic arthritis: we had a few such patients treated with radioactive gold and we were rather impressed with the results tecause they tended to have thin synovial membranes and large effusions; I do not know if anyone else has got any experience with psoriatic arthritis.

DR. SCOTT Of the seventeen patients originally published by Grahame, Ramsey, and myself, five were psoriatics with Baker's cysts and they did well. I must say we have heard some very interesting information today about the escare of radioactivity from the joint. Firstly, Dr. Ramsey and others have made it clear that this is very variable, even when conditions are standardized in terms of the patient's leg being rested and of the substances used (colloidal particle size). Secondly Dr. Goode has mace the very interesting observation that this leakage is largely preventable by pretreatment with hydrocortisone, which makes it look more and more as if leakage is related to the disease activity in the joint. I do not know if anyone has been able to make any observations of this; it is very difficult to make objective 
estimates and it may well be that Dr. Yates will be able to correlate his histology with the degree of escape in due course, but it looks as if disease activity is the main factor in escape.

DR. GOODE Most of my patients treated with ${ }^{198} \mathrm{Au}$ had received myocrisin beforehand, and the knees treated were the joints with persistent trouble. I did not pick out patients with prominent knee effusions, treat them first with ${ }^{198} \mathrm{Au}$ and then perhaps use systemic gold at a later stage. This may have influenced my results, but I have yet to assess how well they have done, and will probably find that gold is not as good as yttrium anyway.

DR. SCOTT Dr. Gumpel and others have looked at models of animals. Have you studied escape from normal joints and from joints with experimental arthritis?

DR. GUMPEL Only in terms of the Table that I showed, which was based on very small numbers. There is an interesting suggestion that the normal knee did retain two of the three colloids considerably better than the inflamed knee, and somebody asked whether I had managed to have the knees at rest. The rabbits were in cages and were placid, but they were not at rest. I am not sure how well we can relate animal models to what happens in humans, as we are not entirely convinced that the animal model is comparable to the patient with rheumatoid arthritis and you do not often find the same amount of fibrin formation in the rabbit.

DR. WILLIAMS Going back one stage to the use of technetium in assessment, there has been a recent report from Scandinavia (Karjalainen, Kettunen, and Holopainen, 1972) of success in deriving an index of uptake of pertechnetate in the knee and using this for assessing treatment by drugs. Might it not be a good idea to test patients before and after treatment, to assess both degree of inflammation beforehand and the success of treatment, and to see whether the latter is related to the former.

DR. ANSELL Some people in Great Britain are not very happy about the use of technetium scans in the assessment of drug therapy over a prolonged period. DR. VEALL The short answer is that $I$ hold no brief for technetium scans one way or the other.

PROF. INGRAND During this meeting varied data and interpretations have been presented, and these may have led to some confusion. We have heard about lymphatic spreading and the factors which may influence it: size of colloid particles, status of the synovium, splinting, and pretreatment with hydrocortisone. Unfortunately these studies have not all been performed in the same conditions, and they are therefore not all comparable.

I think it necessary to point out certain important features so that the participants in this meeting do not go away with the idea that there is no solution to our problems.
In the first place, it is better to use colloidal particles of at least $50 \mathrm{~nm}$. in diameter with a firmly adsorbed $\beta$-ray emitter, the energy of which is compatible with the nature and thickness of the synovium.

The second important thing is the degree of impairment of the joint. Hydrocortisone pretreatment and splinting seem to be the only means of reducing what can be reduced.

I must also give two last items of information to rheumatologists who deal with the injection of radiocolloids.

Firstly, it should be remembered that leakage of radioactivity from the injected joint into the body may occur not only from the knee but also from other joints such as the elbow and wrist. An example of the latter is shown in the Figure.

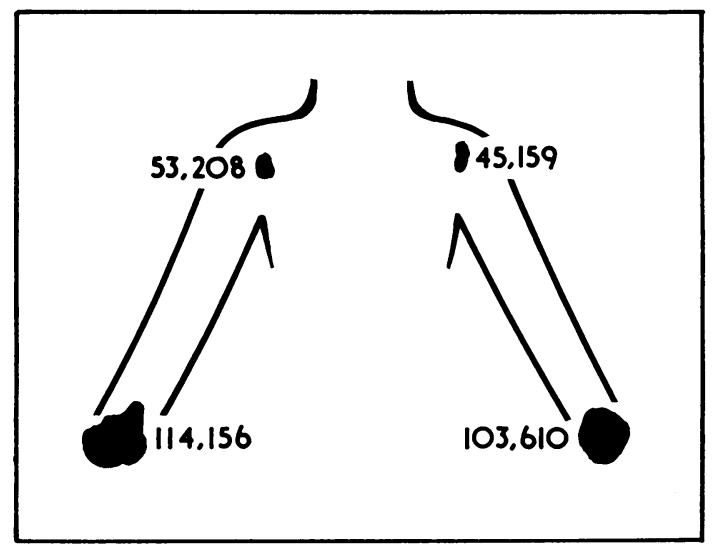

FIGURE Lymphatic spreading of radioactivity after the injection of ${ }^{198} \mathrm{Au}$ into the wrist joint

Secondly, the rheumatologist-even if he is well trained in administering radio-isotope injectionsmay himself absorb a certain amount of radioactivity. By placing small thermoluminescent dosimeters inside his gloves, we were able to measure the dose absorbed on the index fingers and thumbs of such a man during one week's work. With the aid of a technician, he carried out a series of 32 injections totalling $48 \mathrm{~m} \mathrm{Ci}$., using ${ }^{198} \mathrm{Au},{ }^{169} \mathrm{Er}$, and ${ }^{90} \mathrm{Y}$, often with $x$-ray amplification. The results may be tabulated as follows:

\begin{tabular}{|c|c|c|}
\hline \multicolumn{2}{|l|}{ Site } & \multirow{2}{*}{$\begin{array}{l}\text { Dosage (m.rem.) } \\
200 \\
650\end{array}$} \\
\hline Right thumb & $\begin{array}{l}\text { Nail } \\
\text { Pulp }\end{array}$ & \\
\hline Right index finger & $\begin{array}{l}\text { Nail } \\
\text { Pulp }\end{array}$ & $\begin{array}{l}250 \\
830\end{array}$ \\
\hline Left thumb & $\begin{array}{l}\text { Nail } \\
\text { Pulp }\end{array}$ & $\begin{array}{l}350 \\
780\end{array}$ \\
\hline Control dosimeter & & 150 \\
\hline
\end{tabular}


The dosage of radioactivity received by the technician in the course of preparation of the isotope, handling syringes, and initial nursing would be approximately the same.

Now the maximum permissible dosage for the hands and forearms is 15 rem. in 3 months, or about 1,200 m.rem in a week. The maximum permissible irradiation for a worker in this field is 6,200 m.rem. These data show that the amount of radioactivity absorbed by the medical staff is well within the safety margin, and will help to deter-

\section{Afterthoughts}

\section{J. M. GUMPEL}

It is clear from the discussion that there is general acceptance of the efficacy of radiocolloids in the treatment of chronic synovitis, and general agreement about the indications for treatment and the selection of patients. Although one suitable controlled study has confirmed the beneficial use of ${ }^{90} \mathrm{Y}$ in the knee, there is still a need for further controlled studies of the treatment of other joints, and a need to clarify the effect of certain other factors such as the previous removal of fibrin from the joint.

Interest is at present focused on two main points: the possibility of long-term hazards of treatment, and the leakage of radio-pharmaceuticals from the joint. These two points are linked, as the long-term hazards seem to be related more to whole body irradiation than to irradiation of the synovium. As a mine how many patients can safely be treated each week.

DR. ANSELL I think we have had an extremely interesting day. We have learned that there is still an enormous field for further work in the use of these isotopes-to find the best type of isotope for each joint and to carry out controlled studies of the use of the many techniques that are now available to us. I am sure that further excellent work will be done and that we shall have to re-convene in a year or two's time to see how we are all getting on. result, the measurement of the retention of radiocolloids in the joint becomes a valuable research procedure rather than an academic exercise, and should certainly replace the measurement of extraarticular spread with its potential 20 per cent. "dead space". The adoption of any measure that will reduce irradiation of other structures is important, and amongst these are the avoidance of radiocolloids with significant $\gamma$ emission, the evaluation of the available radiocolloids to compare their relative retention and common patterns of spread, and probably most important of all the investigation of the variable permeability of the individual synovial membrane, and the application of methods of reducing transport across the membrane, as for example by rest and by premedication. 